\title{
Le conseguenze sulla spesa \\ farmaceutica pubblica di un nuovo inibitore di pompa protonica: esomeprazolo
}

\author{
C. Lucioni ${ }^{*}$, S. Mazzi ${ }^{*}$, C. Negrini ${ }^{\circ}$
}

\begin{abstract}
Aim of the present study is to check the economic advantage of esomeprazole, a new proton pump inhibitor that suppresses gastric acid secretion. The efficacy and tolerability of esomeprazole have been already demonstrated, now the pharmacoeconomic studies must investigate the possible savings for the SSN in case of esomeprazole immission on the Italian market.

In this paper the authors present two pharmacoeconomic evaluations, the first based on cost/efficacy analysis, the second based on cost minimization analysis. For both analyses, the term of comparison is omeprazole, "gold standard" in the treatment of acid reflux-related pathologies.

The effect in terms of volumes (DDD) and costs of the introduction of esomeprazole in the italian market has also been simulated.
\end{abstract}

Farmeconomia e percorsi terapeutici 2002; 3 (1): 5-14

\section{PREMESSA}

Esomeprazolo (il principio attivo di NEXIUM () è un nuovo farmaco inibitore della pompa protonica (IPP), specifico dunque nel trattamento delle patologie acido-correlate.

Esomeprazolo ha dimostrato superiore efficacia rispetto agli altri IPP, mantenendo il già valido profilo di tollerabilità proprio della classe cui appartiene.

Il presente studio si propone di verificare in quale misura esomeprazolo, avendo già le carte in regola sul piano dell'efficacia e della tollerabilità clinica, possa dimostrarsi valido anche in termini di convenienza economica. Quest'ultima viene qui intesa e misurata come risparmio eventualmente realizzabile dal Servizio Sanitario Nazionale (SSN) in seguito all' introduzione di esomeprazolo sul mercato italiano.

Lo studio si apre con due analisi di valutazione farmacoeconomica, una di costo/efficacia, l'altra di minimizzazione dei costi, in cui esomeprazolo viene confrontato con omeprazolo, "gold standard" nella terapia delle patologie acido-correlate.

\section{ANALISI COSTO/EFFICACIA DI ESOMEPRAZOLOVERSUS OMEPRAZOLONELTRATTAMENTO DELL'ESOFAGITEEROSIVADA REFLUSSO}

In due studi clinici condotti negli Stati Uniti - multicentrici, randomizzati, in doppio cieco, su pazienti $H$-pylori negativi (alla sierologia) e affetti da esofagite erosiva fotodocumentata è stata valutata l'efficacia di esomeprazolo rispetto a quella di omeprazolo nel trattamento dell'esofagite erosiva da reflusso.

Nel primo studio, 1.216 pazienti sono stati trattati fino a otto settimane con esomeprazolo $40 \mathrm{mg}$ al giorno (qd, quaque die) e altri 1.209 con omeprazolo $20 \mathrm{mg}$ qd (Richter et al., 2000).

L'indicatore primario d'efficacia era costituito dalla percentuale di pazienti guariti all'ottava settimana, uno dei secondari dall'analoga percentuale alla quarta settimana. In questi termini, i risultati sono riportati nello schema seguente.

\author{
* ADIS International, \\ Milano \\ - Economic Affairs, \\ AstraZeneca Italia
}




\begin{tabular}{|lccc|}
\hline & $\begin{array}{c}\text { Esomeprazolo } \\
40 \mathrm{mg}\end{array}$ & $\begin{array}{c}\text { Omeprazolo } \\
20 \mathrm{mg}\end{array}$ & $\mathrm{p}$ \\
\hline $\begin{array}{l}\text { Guarigione a } \\
8 \text { settimane }\end{array}$ & $93,7 \%$ & $84,2 \%$ & $<0,001$ \\
\hline $\begin{array}{l}\text { Guarigione a } \\
4 \text { settimane }\end{array}$ & $81,7 \%$ & $68,7 \%$ & $<0,001$ \\
\hline
\end{tabular}

In fatto di tollerabilità, peraltro, le percentuali di pazienti colpiti da eventi avversi collegabili al trattamento nei due rispettivi bracci sono risultate sovrapponibili.

Nel secondo studio, 654 pazienti hanno ricevuto esomeprazolo $40 \mathrm{mg}$ qd, 656 esomeprazolo $20 \mathrm{mg}$ qd, 650 omeprazolo $20 \mathrm{mg}$ qd (Kahrilas et al., 2000).

Gli indicatori di efficacia erano gli stessi già visti nello studio precedente; in particolare, relativamente ai trattamenti con esomeprazolo 40 mg e con omeprazolo $20 \mathrm{mg}$., si sono avuti i risultati riportati nella tabella seguente.

\begin{tabular}{|lccc|}
\hline & $\begin{array}{c}\text { Esomeprazolo } \\
40 \mathrm{mg}\end{array}$ & $\begin{array}{c}\text { Omeprazolo } \\
20 \mathrm{mg}\end{array}$ & $\mathrm{p}$ \\
\hline $\begin{array}{l}\text { Guarigione a } \\
8 \text { settimane }\end{array}$ & $94,1 \%$ & $86,9 \%$ & $<0,05$ \\
\hline $\begin{array}{l}\text { Guarigione a } \\
4 \text { settimane }\end{array}$ & $75,9 \%$ & $64,7 \%$ & $<0,05$ \\
\hline
\end{tabular}

Tutti gli indicatori sopra riportati sono confluiti nella Tabella $A$ allegata, che è alla base di questa prima analisi; nella stessa tabella, inoltre, in vista dei calcoli da compiere per la valutazione, i rispettivi dati di efficacia provenienti dai due studi sono stati aggregati facendone delle medie ponderate col numero dei pazienti trattati, così da avere - per ciascun principio attivo considerato - un solo dato di efficacia a otto settimane e uno solo a quattro.

Passando al versante dei costi, il prezzo dei farmaci è quello vigente al 30 marzo $2002^{\circ}$. Si è applicato a esomeprazolo il prezzo al pubblico recentemente negoziato (Gazzetta Ufficiale, 2002) di a 31,61 per la confezione di 14 comrisultati dei confronti del presente studio.

Figura 1 presse da $40 \mathrm{mg}$; per omeprazolo, si adotta il prezzo al pubblico attualmente (marzo 2002) in vigore per le specialità con confezione di 14 compresse da $20 \mathrm{mg}$ : $\square$ 33,1 (L'Informatore Farmaceutico, 2002). Ne deriverebbe un costo di trattamento per otto settimane con i due principi attivi, rispettivamente, di ud 26,44 e di $\mathrm{1}$ 132,4.

Occorre però tenere presente che il trattamento acuto non viene protratto a otto settimane su tutti i pazienti, bensì soltanto su quelli che non risultano ancora guariti all'esame endoscopico effettuato dopo quattro settimane; per gli altri, il trattamento acuto è limitato a quattro settimane, e così il relativo costo. Il corretto costo medio di trattamento si ottiene dunque sottraendo dal costo pieno calcolato su otto settimane il costo di trattamento per quattro settimane moltiplicato per la probabilità che questo non debba essere sostenuto (costo di trattamento evitato), in quanto il paziente risulti già guarito dopo le prime quattro settimane di trattamento. Tale probabilità non è altro che la percentuale media ponderata di efficacia clinica a quattro settimane $(79,7 \%$ per esomeprazolo e $67,3 \%$ per omeprazolo).

In definitiva, come mostra la Tabella $A$, il costo medio effettivo di trattamento risulta pari a $\square 76,06$ per esomeprazolo e $\square 87,65$ per omeprazolo.

A questo punto, il trattamento con esomeprazolo risulta strategia dominante, in quanto presenta un tasso di efficacia superiore, unito a un costo, per paziente trattato e per paziente guarito, inferiore a quello del farmaco di confronto, come evidenziato in Figura 1.

Nell'esofagite erosiva da reflusso, dunque, con i dati di costo e d'efficacia considerati sopra, esomeprazolo si pone in ogni caso come farmaco economicamente conveniente rispetto ad omeprazolo.

\section{ANALISIDI MINIMIZZAZIONE DEI COSTI DIESOMEPRAZOLO VSOMEPRAZOLO NELL'ERADICAZIONE DELL'HELICOBACTER PYLORI E NELLA GUARIGIONEDELL'ULCERA DUODENALE}

Due studi multicentrici internazionali, randomizzati, in doppio cieco, hanno confrontato l'efficacia di esomeprazolo con quella di omeprazolo nella terapia eradicante dell' $H$ pylori e dell'ulcera duodenale.

Il primo includeva pazienti $H$-pylori positivi e con ulcera duodenale attiva (Tulassay et al., 2000). Di essi, 222 sono stati trattati con esomeprazolo $20 \mathrm{mg}$ due volte al giorno (bid, bis in die) in combinazione con amoxicillina $1 \mathrm{~g}$ bid e claritromicina $500 \mathrm{mg}$ bid; 224 sono stati trattati con omeprazolo $20 \mathrm{mg}$ bid e con la me- 


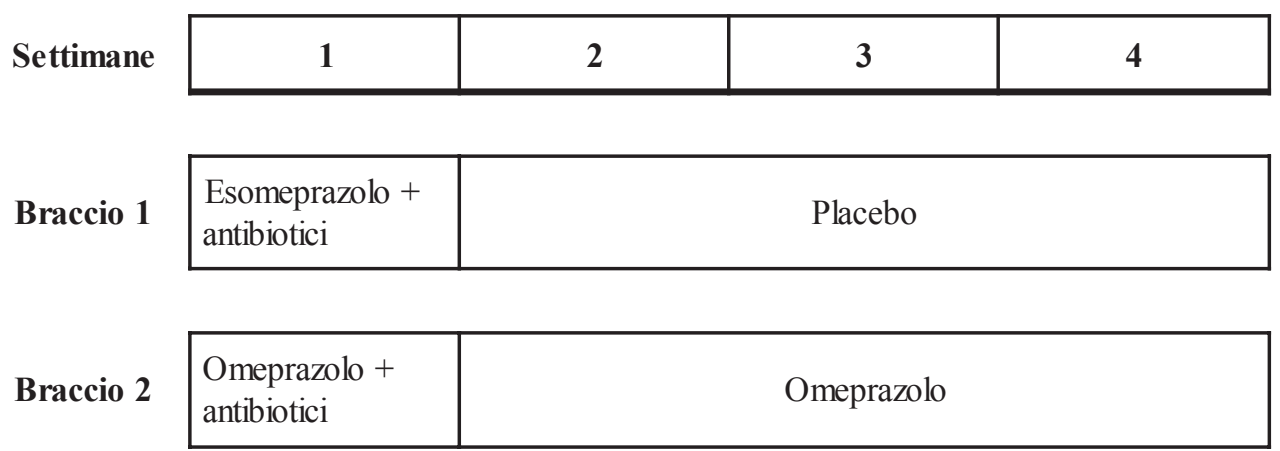

Schema 1

(da Tulassay et al. 2000) desima combinazione di antibiotici.

Nel braccio di esomeprazolo il trattamento è durato una sola settimana, seguita da tre settimane in cui è stato somministrato placebo. Nel braccio di omeprazolo, invece, il trattamento con l'inibitore di pompa si è protratto per quattro settimane, come rappresentato nello Schema 1.

La percentuale di guarigione dall'ulcera è stata del $91 \%$ nel braccio di esomeprazolo e del 92\% nell'altro; quella di eradicazione dell' $H$ pylori è stata dell' $86 \%$ nel primo e dell' $88 \%$ nel secondo; nessuna differenza di efficacia era, comunque, statisticamente significativa.

Nel secondo studio, condotto su 448 pazienti $H$-pylori positivi e con ulcera duodenale non attiva, la metà dei pazienti è stata trattata per una settimana con la triplice terapia già vista sopra a base di esomeprazolo, l'altra metà ugualmente per una settimana - con la triplice terapia a base di omeprazolo (Veldhuyzen et al., 2000). Le percentuali di eradicazione dell' $H$ pylori nei due bracci - rispettivamente dell' $89,7 \%$ e dell' $87,8 \%$ - non sono risultate significativamente differenti.

La conclusione che si trae da questi due studi è che esomeprazolo è efficace quanto omeprazolo, ai dosaggi e nelle combinazioni indicate, nell'eradicazione dell' $H$-pylori e nella guarigione dell'ulcera duodenale attiva. Il confronto di convenienza economica tra i due principi attivi si traduce così nella ricerca di quello col minor costo.

Di fatto, il vantaggio clinico ed economico di esomeprazolo è rappresentato dalla durata del trattamento.

Gli inibitori di pompa di "vecchia generazio- ne" sono stati impiegati di norma secondo uno schema posologico che ne prevedeva la somministrazione per quattro settimane (la prima delle quali in associazione con due antibiotici).

Esomeprazolo è il primo inibitore in possesso di un'indicazione specifica, che ne consente l'impiego per una sola settimana (naturalmente in combinazione con due antibiotici), per ottenere sia l'eradicazione dell' H-pylori che la guarigione dell'ulcera ad esso associata.

Adottando per una confezione di esomeprazolo di 14 compresse da $20 \mathrm{mg}$ il prezzo al pubblico di $\bullet 24,38$ (Gazzetta Ufficiale, 2002) e per gli altri farmaci i prezzi al pubblico attuali (si veda la Tabella $B$ allegata), nel trattamento dell'ulcera duodenale con eradicazione dell' $H$ pylori il costo per paziente trattato con la combinazione di farmaci facente capo a esomeprazolo risulta essere di • 77,62 mentre quello del farmaco di confronto ammonta a 135,99 . Ciò significa che l'adozione del trattamento con esomeprazolo in luogo di quella con omeprazolo permetterebbe di realizzare un risparmio del $43 \%$, pari cioè a quasi la metà del costo di quest' ultimo.

Come invece mostra la Tabella $C$, il risparmio è del $10 \%$ quando l'indicazione terapeutica e il relativo trattamento sono limitati all'eradicazione dell'H-pylori.

\section{DUESCENARIPERILMERCATODEGLI IPPIN ITALIANELPROSSIMOFUTURO}

Il mercato degli IPP in Italia è stato in fase di continua espansione nel corso degli ultimi anni, raggiungendo nel 2001 un valore (a prezzi al

\section{Nota ii}

La DDD (Defined Daily Dose, dose giornaliera definita) equivale all'ipotetica dose media giornaliera di un farmaco impiegato nel trattamento di un adulto di $70 \mathrm{~kg}$. con riferimento all'indicazione terapeutica principale (Clarke et al., 1995). Anche se tiene conto della prassi clinica e delle indicazioni delle Case produttrici, la DDD non è propriamente né una media statistica né una posologia particolarmente raccomandata; essa è tipicamente uno standard di misura dei consumi farmacologici. Punto di riferimento internazionale per la metodologia delle DDD e i relativi dati è il WHO Collaborating Centre for Drug Statistics Methodology (WHO, 2000). Per il mercato italiano, la fonte dati delle DDD è la sezione italiana del Drug Utilisation Research Group (DURG-Italia, 1999). 
pubblico) di circa 600 milioni di Euro. Il suo volume complessivo, misurato in DDD ii, è più che raddoppiato, passando da circa 95 milioni di DDD nel 1995 a 268 nel 2001 iii. In tale periodo, a omeprazolo, capostipite della classe, si sono via via aggiunti altri concorrenti: lansoprazolo (lanciato nel 1995), pantoprazolo (1997), rabeprazolo (1999), complessivamente 101 milioni di DDD nel 2001 (IMS, vari anni).

Nella Tabella $D$ è fotografata in dettaglio la recente (a fine 2001) situazione del mercato degli IPP in Italia. Quest'ultimo è espresso in termini di volumi (unità vendute), valori (Euro) e DDD, così da poter avere dati a quantità perfettamente confrontabili e aggregabili.

Da quella tabella risulta che l'attuale dimensione del mercato degli IPP, a valori ammonta a circa 600 milioni di Euro. La disponibilità terapeutica totale è di circa 268 milioni di DDD; il costo di $\bullet 2,24$ per DDD rappresenta il costo medio generale per avere un giorno di trattamento con IPP. Uno stralcio sintetico è riportato in Tabella 1.

Ciò premesso, ipotizzando che l'impiego
Tabella 1

Consumo e costo degli IPP in Italia, nel 2001

\section{Tabella 2}

Consumo e costo degli IPP in Italia, nel 2004, senza esomeprazolo

\begin{tabular}{|lccc|}
\hline \multicolumn{1}{|c}{ Prodotti } & DDD (milioni) & quota di mercato & costo/DDD (Euro) \\
\hline Omeprazolo & 167 & $62 \%$ & 2,35 \\
\hline Lansoprazolo & 39 & $15 \%$ & 2,29 \\
\hline Pantoprazolo & 35 & $13 \%$ & 2,01 \\
\hline Rabeprazolo & 27 & $10 \%$ & 1,77 \\
\hline \multicolumn{1}{r}{ Totale } & 268 & $100 \%$ & 2,24 \\
\hline
\end{tabular}

\begin{tabular}{|lccc|}
\hline \multicolumn{1}{|c}{ Prodotti } & DDD (milioni) & Quota di mercato & costo/DDD (Euro) \\
\hline Omeprazolo & 208 & $62 \%$ & 2,35 \\
\hline Lansoprazolo & 50 & $15 \%$ & 2,29 \\
\hline Pantoprazolo & 44 & $13 \%$ & 2,01 \\
\hline Rabeprazolo & 33 & $10 \%$ & 1,77 \\
\hline totale & 335 & $100 \%$ & 2,24 \\
\hline
\end{tabular}

Tabella 3

Consumo e costo degli IPP in Italia, nel 2004, con esomeprazolo

\begin{tabular}{|c|c|c|c|}
\hline Prodotti & DDD (milioni) & Quota di mercato & costo/DDD (Euro) \\
\hline Omeprazolo & 166 & $50 \%$ & 2,35 \\
\hline Altri prodotti & 101 & $30 \%$ & 2,06 \\
\hline Esomeprazolo & 68 & $20 \%$ & 1,55 \\
\hline Totale & 335 & $100 \%$ & 2,1 \\
\hline
\end{tabular}

\section{Nota iii}

In termini approssimativi circa l' $1 \%$ della popolazione è ora arrivato ad essere in trattamento con IPP (268 milioni di DDD nell'anno 2001 corrispondono a circa 12 DDD per 1.000 abitanti al giorno). Valutando che la prevalenza complessiva dei disturbi per patologie acido correlate sia in Italia molto più elevata, si può dire che della popolazione rimanente una parte (corrispondente a circa $0,5 \%$ della popolazione complessiva, ovvero a $5 \mathrm{DDD} / 1.000 \mathrm{ab} . /$ die) è trattata con $H$ antagonisti e tutto il resto con sintomatici o non è trattato affatto. Il consumo di IPP in Italia appare modesto se confrontato ${ }^{2}$ con quello di altri paesi europei. In Francia, per esempio, tale consumo si aggira intorno alle 16.5 DDD/1000 ab./ die, mentre nel Regno Unito è pari a 19.3 DDD/1000 ab./ die (elaborazione da dati IMS, anno 2000). 
degli inibitori di pompa protonica in Italia tenda ad approssimarsi a quello degli altri paesi europei, in particolare a quello della popolazione francese, possiamo ipotizzare che nel 2004 il mercato degli IPP in Italia, crescendo con gli attuali prodotti, potrà arrivare a 335 milioni di DDD per un valore di 750 milioni di Euro. Assumendo allora che il mix di mercato e i relativi prezzi rimangano invariati tra il 2001 e il 2004, nel 2004 si renderebbero prevedibilmente disponibili circa 335 milioni di DDD e la situazione sarebbe quella illustrata in Tabella 2.

A questo primo scenario (in cui si prevede che il mercato si sviluppi con solo i principi attivi attualmente esistenti) può esserne affiancato un secondo, ipotizzando invece che sul mercato venga introdotto esomeprazolo (a partire dal 2002). Il confronto tra i due scenari è centrale nel presente lavoro, in quanto consente di mettere in luce le conseguenze potenzialmente derivanti dall'introduzione di esomeprazolo.

Per costruire il secondo scenario è necessario innanzitutto stimare quale potrebbe essere la quota di mercato assunta da esomeprazolo nel $2004{ }^{\text {iv }}$, cioè nel terzo anno di presenza sul mercato; a questo proposito, si possono fare alcune considerazioni.

Al terzo anno dal lancio (1999) la quota di mercato $^{v}$ di pantoprazolo era del 16\%. Nel 2001 (analogamente) la quota di mercato di rabeprazolo era del 10\%. Per esomeprazolo si potrebbe adottare, indicativamente, il valore medio tra questi due dati $(13 \%)$; tuttavia, tenendo conto che il prodotto rappresenta un superamento di omeprazolo (il gold standard tra gli IPP) e che esso mostra (come si è già visto più sopra) notevoli valenze di convenienza economica, appare ragionevole adottare un valore superiore - quale potrebbe essere una quota di mercato del $20 \%$.

In questo caso, ipotizzando che esomeprazolo si espanda sul mercato solo per effetto di sostituzione, riducendo proporzionalmente lo spazio ai prodotti esistenti, il secondo scenario si configurerebbe come mostrato in Tabella 3.

$\mathrm{Ne}$ consegue che il valore complessivo del mercato, ottenuto moltiplicando il costo/DDD medio $(\bullet 2,1)$ per il numero totale delle DDD disponibili (335 milioni), sarebbe di circa 703 milioni di Euro ${ }^{\text {vi }}$. Tale valore rappresenta, d'altra parte, l'onere economico che il SSN sostiene per garantire il trattamento con IPP.

Poiché nello scenario precedente (senza esomeprazolo) l'analogo valore era di $750 \mathrm{mi}-$ lioni di Euro, ciò significa che l'introduzione di esomeprazolo sul mercato potrebbe fare risparmiare al SSN, nel solo 2004, 47 milioni di Euroa parità di DDD disponibili, beninteso.

\section{ANALISIDISENSIBILITÀ}

Trattandosi di una stima, e quindi per esplorare la tenuta delle conclusioni che vi sono legate, abbiamo provato a variare per il $50 \%$ del suo valore in più e in meno la quota di mercato del $20 \%$ prevista per esomeprazolo nel 2004 -e cioè da un minimo del $10 \%$ a un massimo del $30 \%$. Le considerazioni sono suggerite dalla seguente Figura 2: la presenza di esomeprazolo sul mercato genera comunque un risparmio, che aumenta all'aumentare della penetrazione del prodotto stesso: 24 milioni di Euro se la quota di mercato è del 10\%, 70 milioni di Euro se la stessa è del $30 \%$.

E' stata fatta anche un' altra verifica: rispetto all'assunzione di base del secondo scenario, secondo cui il mix di esomeprazolo sarebbe stato composto nel 2004 per il $20 \%$ da confezioni da $40 \mathrm{mg}$ e per l' $80 \%$ da confezioni da 20 $\mathrm{mg}$, tali percentuali sono state fatte variare rispettivamente a $40 \%$ e a $60 \%$. Ciò comporta che il valore complessivo del mercato si collocherebbe a circa 717 milioni di euro; col che, il risparmio consentito dalla presenza di esomeprazolo aggiunto agli attuali prodotti sarebbe di 33 milioni di Euro (14 in meno rispetto a quelli già evidenziati nell' analisi di base).

\section{DISCUSSIONE E CONCLUSIONI}

La verifica di convenienza economica che costituisce l'obiettivo del presente studio è stata condotta iniziando con due analisi, una di costo-efficacia, l'altra di minimizzazione dei costi, nelle quali esomeprazolo veniva confrontato con omeprazolo nel trattamento dell'esofagite erosiva da reflusso e, rispettivamente, nell' eradicazione dell' Helicobacter pylori. Nel primo caso, esomeprazolo risultava strategia

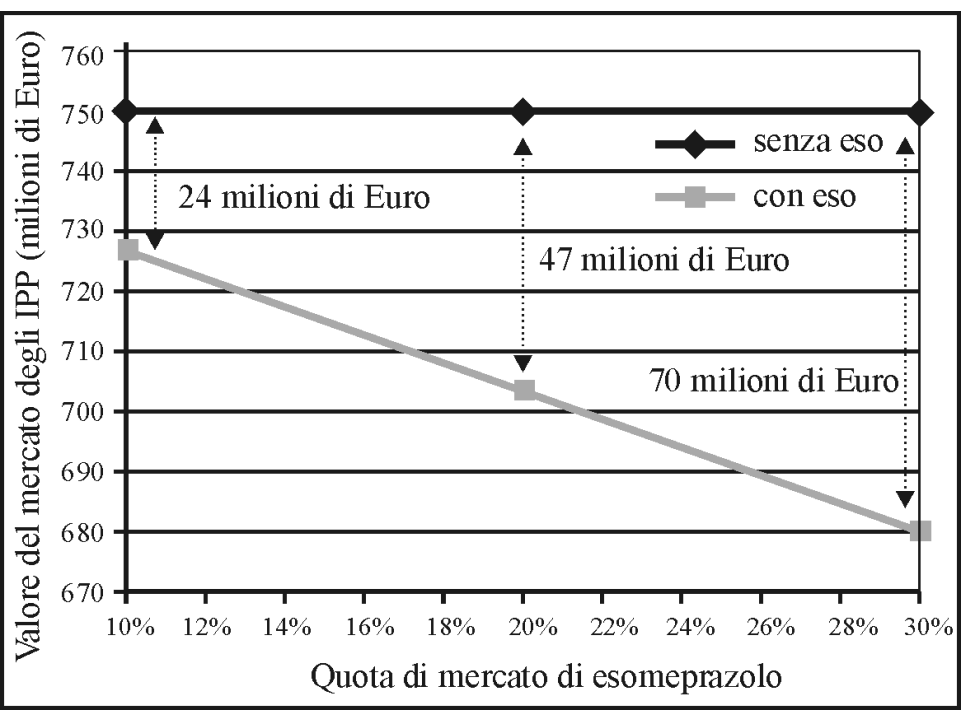

Figura 2
Notaiv

E' stato scelto come anno di riferimento degli scenari il 2004 proprio perché per allora la situazione di esomeprazolo sul mercato dovrebbe ritenersi a regime.

\section{Notav}

Sempre espressa, beninteso, in termini di $D D D$

\section{Nota vi}

Il costo per DDD di esomeprazolo è stato calcolato sulla base dei prezzi al pubblico recentemente negoziati e prevedendo che le confezioni vendute siano - a numero di pezzi - per il $20 \%$ da 40 mg e per l' $80 \%$ da $20 \mathrm{mg}$. 


\section{Nota vii}

Sarebbe però lecito presumere che questa ulteriore crescita dell'offerta di IPP possa avvenire anche a fronte di un ridimensionamento della domanda di $\mathrm{H}$ antagonisti (quindi, in realtà, ancora con un effetto di sostituzione, sia pure su un altro mercato). dominante (cioè più efficace e meno costosa al contempo del farmaco di confronto), nel secondo strategia capace di far conseguire sostanziali risparmi al SSN (fin quasi del $50 \%$ rispetto alla terapia con omeprazolo).

La verifica si è poi rivolta a una prospettiva più generale: quella del mercato complessivo degli IPP in Italia. Sono stati configurati due scenari al 2004 (in prima approssimazione, con mix e prezzi invariati rispetto al 2001), l'uno in cui si ipotizzava l'assenza di esomeprazolo, l'altro in cui veniva assunta l'introduzione di quest'ultimo sul mercato, a partire dal 2002. In questo secondo caso è stata fatta - in mancanza di informazioni che consentissero un approccio più articolato - l'ulteriore ipotesi che la conquista del proprio spazio da parte di esomeprazolo avvenisse solo per effetto di sostituzione (con una riduzione proporzionale delle quote di mercato dei prodotti esistenti) e non anche per effetto di innovazione (cioè con una propria aggiunta netta all'offerta complessiva $^{\text {vii }}$.

$\mathrm{Ne}$ è risultato che nel terzo anno di presenza di esomeprazolo, il SSN conseguirebbe un risparmio (annuo) di spesa di quasi 50 milioni di Euro (grazie al minor costo della DDD del nuovo prodotto rispetto a quello degli attuali); ciò avverrebbe con un pari livello di soddisfacimento della domanda e con un miglioramento delle possibilità terapeutiche dei pazienti affetti da malattie acido correlate, grazie alla maggior efficacia di esomeprazolo rispetto agli IPP concorrenti.

Naturalmente, va tenuto presente che se vi fosse anche un effetto di innovazione esercitato da esomeprazolo, vi sarebbe coeteris paribus un minore risparmio per il SSN, in quanto quest'ultimo dovrebbe pagare per un mercato di dimensioni maggiori - mercato che consentirebbe peraltro anche una più ampia disponibilità di IPP.

Fermi restando i limiti della semplificazione adottata, l'analisi di sensibilità ha poi confermato che il risparmio realizzabile dal SSN aumenta con l'aumentare della quota di mercato conquistata da esomeprazolo ai prodotti concorrenti.

In considerazione di tutto ciò, sembra di poter concludere che l'introduzione di esomeprazolo sul mercato italiano si prospetta con connotati di positività sotto il profilo della convenienza economica, oltre che sotto il profilo dell'efficacia.

Questo studio è stato reso possibile da un finanziamento messo a disposizione da AstraZeneca S.p.A.

\section{BIBLIOGRAFIA}

Clarke K.W., Gray D. - The Defined Daily Dose as a tool in Pharmacoeconomics. Advantages and limitations PharmacoEconomics 7 (4), 280-283, 1995

Drummond M.F., O’Brien B.J., Stoddart G.L., Torrance G.W. - Metodi per la valutazione economica dei programmi sanitari. Seconda edizione - Edizione italiana a cura di G. Fattore, L. Garattini, C. Lucioni, Il Pensiero Scientifico Editore, Roma, 2000

DURG-Italia - Archivio delle DDD dei farmaci in commercio in Italia - Bologna, settembre 1999

Gazzetta Ufficiale n ${ }^{\circ} 30$ del 5.02.2002, Decreto AIC/UAC n ${ }^{\circ} 649$ del 3.01.2002

Gold M.R., Siegel I.E., Russell L.B., Weinstein M.C. (eds.) - Cost-effectiveness in health and medicine - Oxford University Press, New York, 1996

Kahrilas P.J., Falk G., Whipple J., D’Amico J., Joelsson B. - Comparison of esomeprazole, a novel PPI, vs omeprazole in GERD patients with erosive esophagitis - Gastroenterol 2000, 118, A93

IMS - Il Mercato Farmaceutico - vari anni

IMS, Servizio Prescrizioni Mediche, 2000

L'Informatore Farmaceutico, 2002 - OEMF, Milano, 2002

Richter J.E., Kahrilas P.J., Hwang C., Marino V., Hamelin B. - Esomeprazole is superior to omeprazole for the healing of erosive esophagitis in GERD patients - Gastroenterol 2000, 118, A20

Tulassay Z., Kryszewski A., Dite P. - 7-day treatment with esomeprazole-based triple therapy eradicates H pylori and heals patients with duodenal ulcer disease - Gastroenterol 2000, 118, A502

Veldhuyzen van Zante S.J.O., Lauristen K., Delchier J.C. - 7-day triple therapy with esomeprazole, amoxicillin and clarithromycin for H pylori eradication in duodenal ulcer disease - Gastroenterol 2000, 118, A503

WHO Collaborating Centre for Drug Statistics Methodology - Guidelines for ATC classification and DDD assignement -Oslo, 2000 


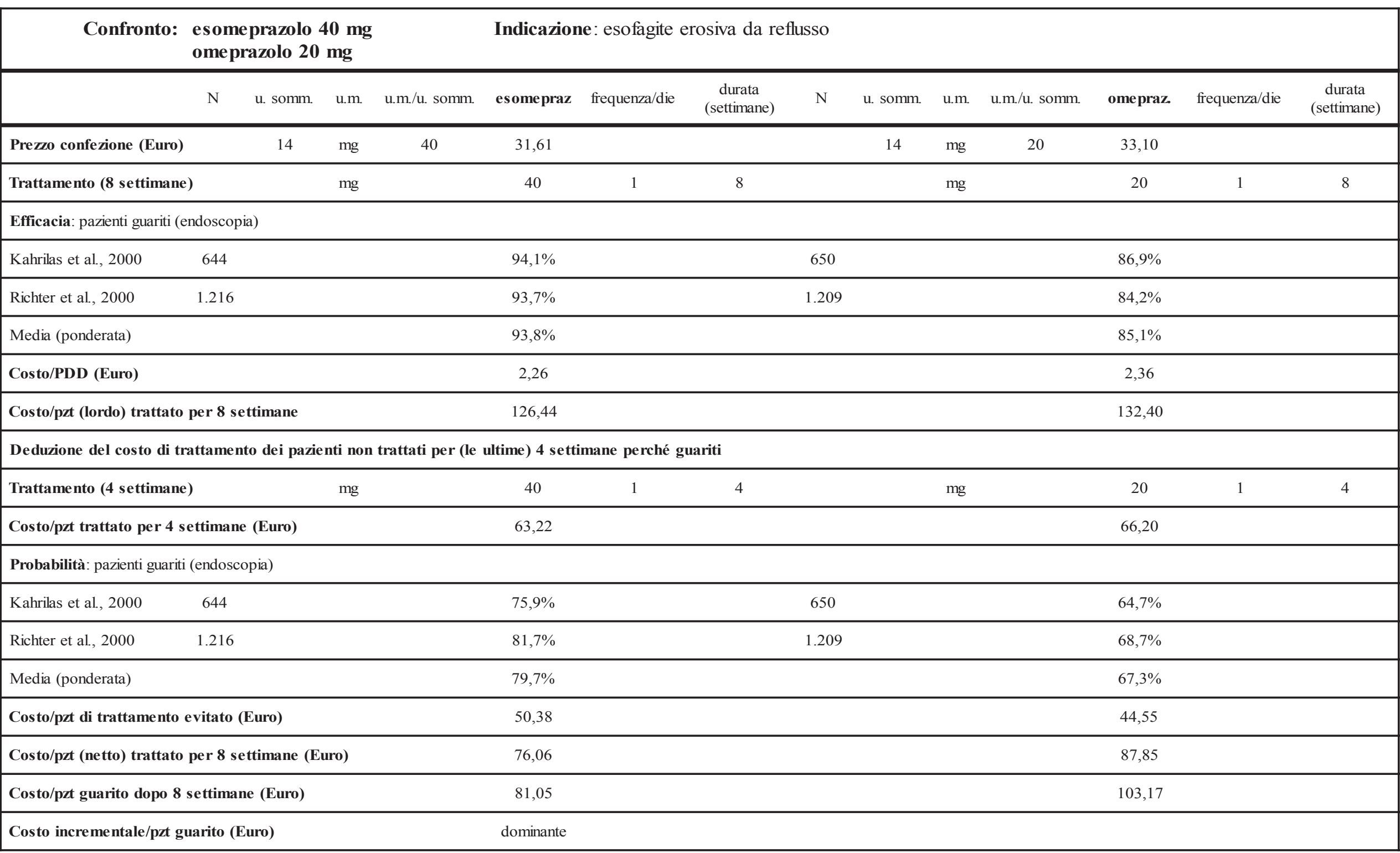

\section{TabellaA}




\section{Tabella B}

Analisi di minimizzazione dei costi

Indicazione: ulcera duodenale con eradicazione H.P.

omeprazolo $20 \mathrm{mg}$

omeprazolo

esomeprazolo

u. somm. u.m. u.m/u.somm. $\begin{gathered}\text { prezzo } \\ \text { confezione } \\ \text { (lire) }\end{gathered}$ frequenza/die $\begin{gathered}\text { durata } \\ \text { (settimanale) }\end{gathered}$

somm. u.m. u.m/u.somm.

prezzo

frequenza/die durata

(lire)

(ire)

\begin{tabular}{|c|c|c|c|c|c|c|c|c|c|c|c|c|c|c|}
\hline $\begin{array}{l}\text { Prezzo } \\
\text { confezione }\end{array}$ & Esomeprazolo & 14 & $\mathrm{mg}$ & 20 & 24,38 & & & Omeprazolo & 14 & $\mathrm{mg}$ & 20 & 33,10 & & \\
\hline & Amoxicillina & 12 & g & 1 & 5,84 & & & Amoxicillina & 12 & $\mathrm{~g}$ & 1 & 5,84 & & \\
\hline & Claritromicina & 14 & $\mathrm{mg}$ & 500 & 46,43 & & & Claritromicina & 14 & $\mathrm{mg}$ & 500 & 46,43 & & \\
\hline Trattamento & Esomeprazolo & & $\mathrm{mg}$ & & 20 & 2 & 1 & Omeprazolo & & $\mathrm{mg}$ & & 20 & 2 & 1 \\
\hline & Amoxicillina & & g & & 1 & 2 & 1 & Amoxicillina & & g & & 1 & 2 & 1 \\
\hline & Claritromicina & & $\mathrm{mg}$ & & 500 & 2 & 1 & Claritromicina & & $\mathrm{mg}$ & & 500 & 2 & 1 \\
\hline & & & & & & & & Omeprazolo & & $\mathrm{mg}$ & & 20 & 1 & 3 \\
\hline
\end{tabular}

Efficacia

efficacia simile (differenze non significative)

Tulassay et al., 2000

Costo/pzt trattato (euro)

77,62

135.99

risparmio-> $43 \%$ 


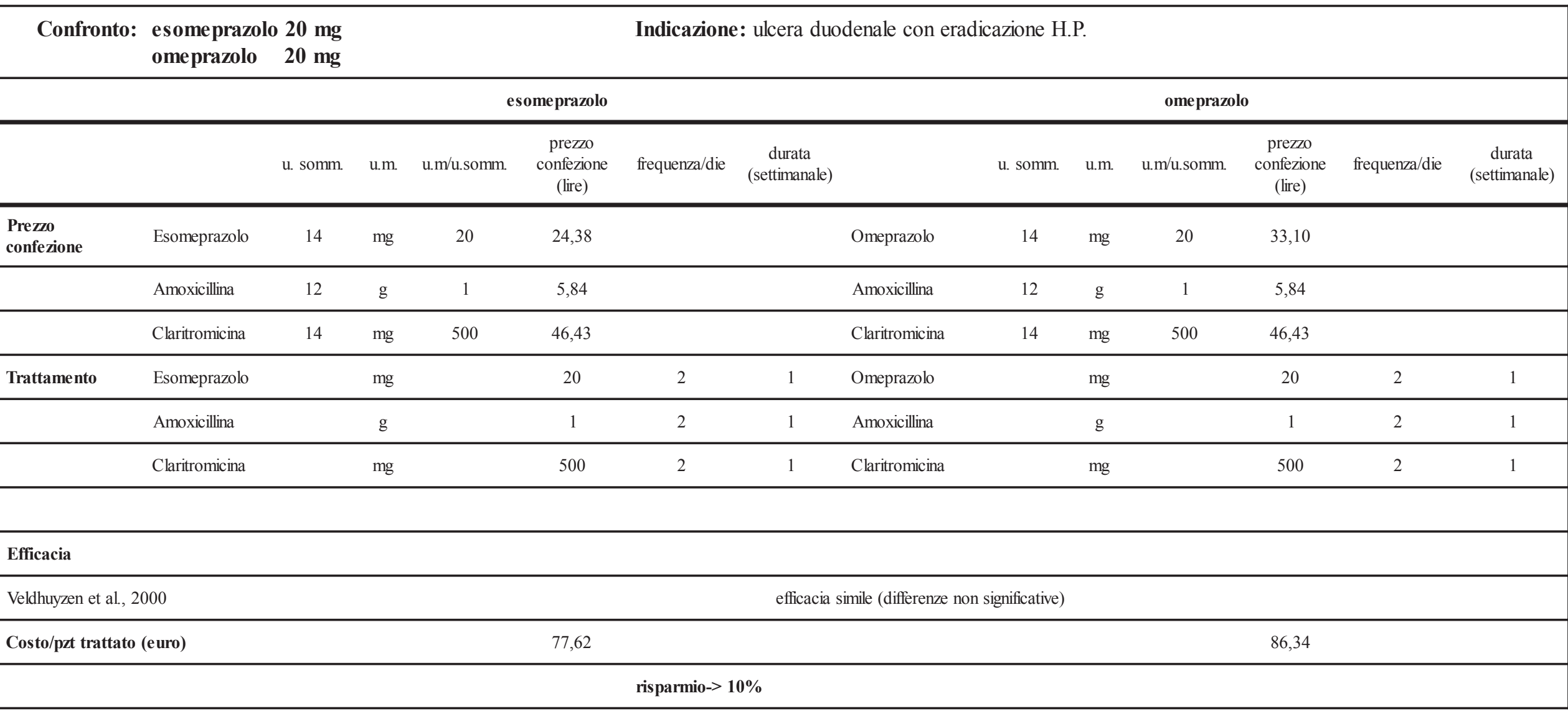

\section{Tabella C}




\begin{tabular}{|c|c|c|c|c|c|c|c|c|}
\hline Principio attivo & Unità di misura & DDD & $\mathrm{N}^{\circ}$ unità/confezione & UM/unità & Prezzo al pubblico (Euro) & $\mathrm{N}^{\circ}$ pezii & DDD & Euro \\
\hline \multirow[t]{2}{*}{ Omeprazolo } & $\mathrm{mg}$ & 20 & 14 & 20 & 33,1 & 10.660 .000 & 149.240 .000 & 352.846 .000 \\
\hline & & 20 & 14 & 10 & 15,65 & 2.500 .000 & 17.500 .000 & 39.125 .000 \\
\hline totale & & & & & & 13.160 .000 & 166.740 .000 & 391.971.000 \\
\hline \multirow[t]{2}{*}{ Lansoprazolo } & $\mathrm{mg}$ & 30 & 14 & 30 & 30,42 & 1.386 .000 & 19.404 .000 & 42.162 .120 \\
\hline & & 30 & 14 & 15 & 16,89 & 2.768 .000 & 19.376 .000 & 46.751 .520 \\
\hline totale & & & & & & 4.154 .000 & 38.780 .000 & 88.913.640 \\
\hline \multirow[t]{2}{*}{ Pantoprazolo } & $\mathrm{mg}$ & 40 & 14 & 40 & 28,25 & 1.980 .000 & 27.720 .000 & 55.935 .000 \\
\hline & & 40 & 14 & 20 & 13,94 & 1.107 .000 & 7.749 .000 & 15.431 .580 \\
\hline totale & & & & & & 3.087 .000 & 35.469 .000 & 71.366 .580 \\
\hline \multirow[t]{2}{*}{ Rabeprazolo } & $\mathrm{mg}$ & 20 & 14 & 20 & 24,38 & 1.595 .000 & 22.330 .000 & 38.886 .100 \\
\hline & & 20 & 14 & 10 & 13,27 & 642.000 & 4.494 .000 & 8.519 .340 \\
\hline totale & & & & & & 2.237 .000 & 26.824.000 & 47.405.440 \\
\hline \multirow[t]{2}{*}{ Totale } & & & & & & 22.638 .000 & 267.813 .000 & 599.656 .660 \\
\hline & & & & & & \multicolumn{2}{|c|}{ costo medio della DDD (euro) -> } & 2,24 \\
\hline
\end{tabular}

\section{Tabella D}

Il mercato degli IPP in Italia, nel 2001 